\title{
Search for new physics
}

with long-lived particles

Christopher Marino, Indiana University

For the ATLAS Collaboration

SUSY09

June 6, 2009

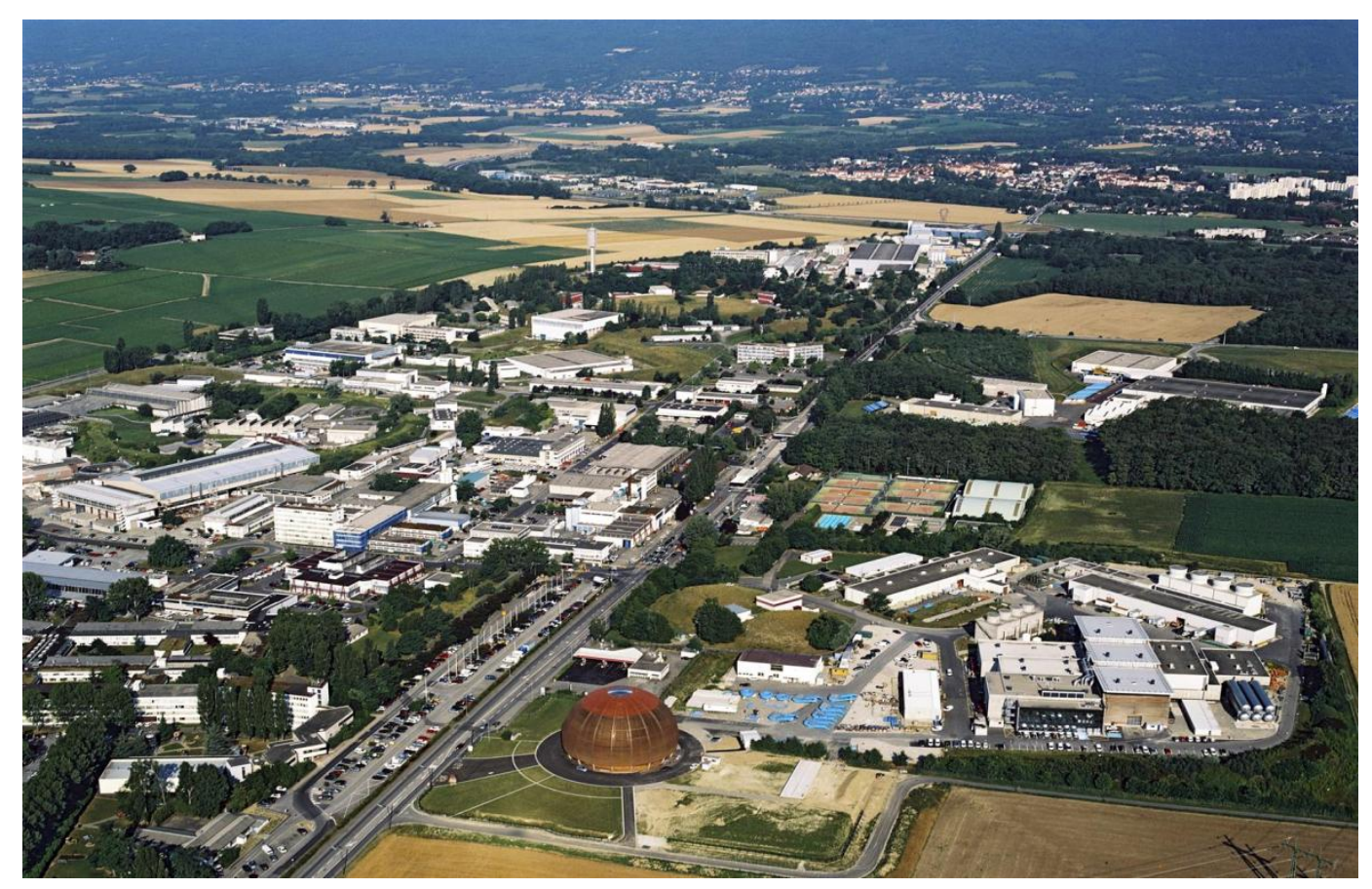




\section{Introduction}

- Various models of new physics predict long-lived particles which travel a significant distance from the interaction point before decaying

- Strategies capitalizing on the unique signatures improve triggering and reconstruction for such events at ATLAS

- This talk will focus on Hidden Valley particles produced through Higgs decays 


\section{Outline}

- Hidden Valley Scenario

- Experimental Challenges

- ATLAS Trigger

- Decays within the Detector

- Muon Spectrometer

- Calorimeter

- Inner Detector

- Status and Plans

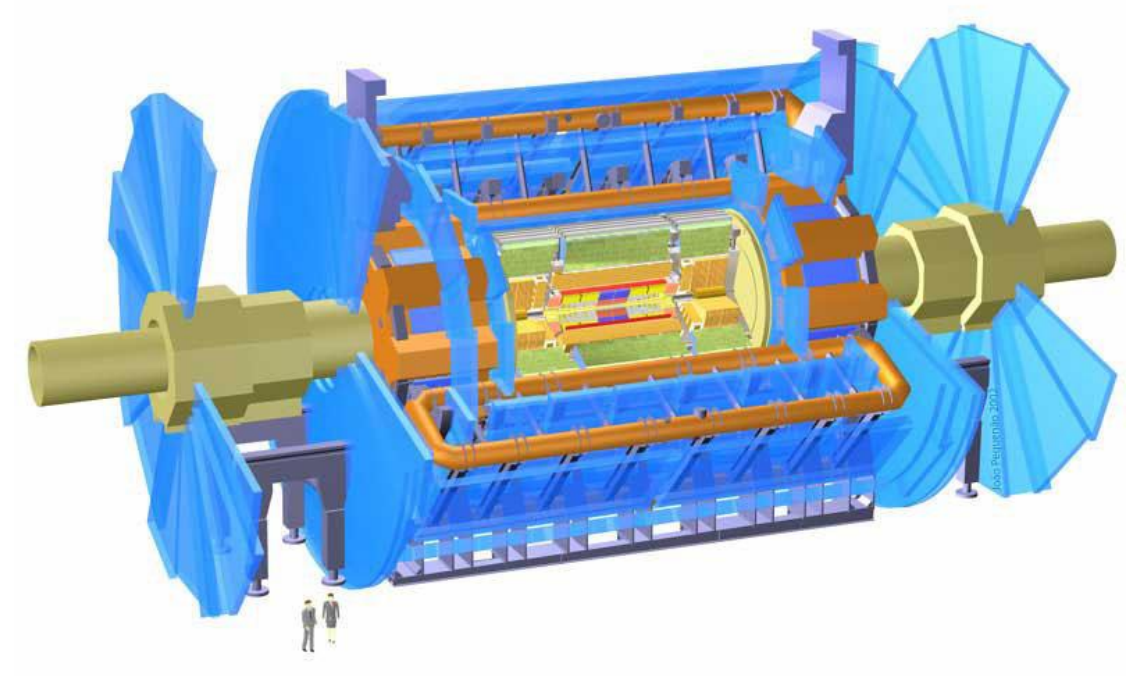




\section{Hidden Valley ${ }^{\dagger}$ Scenarios}

- Beyond the Standard Model is

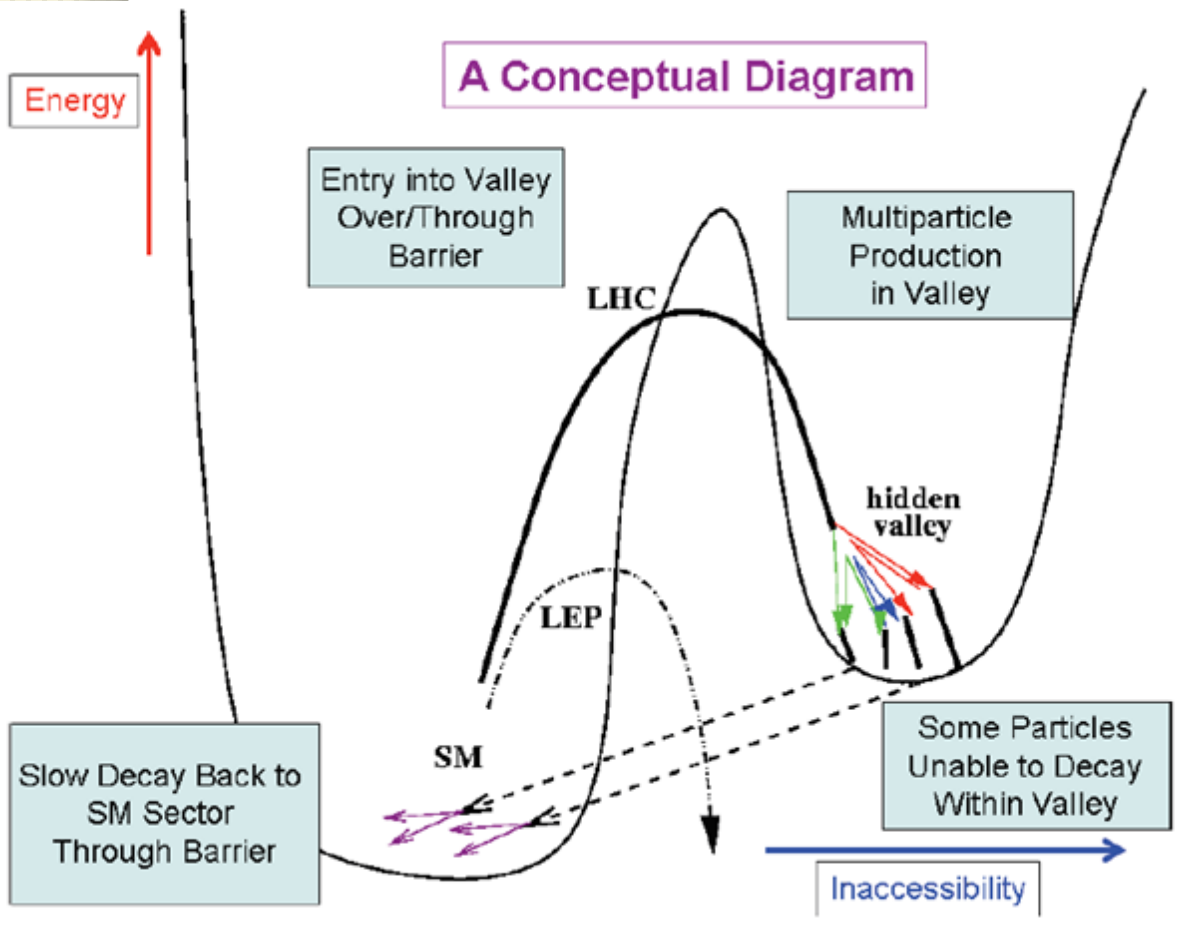

†see:

M. Strassler, K. Zurek Echoes of a Hidden Valley at Hadron Colliders, Phys. Lett. B65I (2007) 374-379

Arkani-Hamed, et al. LHC signals for a SuperUnified

Theory of Dark Matter, arXiv:0810.07I3 a hidden sector (or v-sector) and a communicator interacts with both sectors

- A barrier "hides" the v-sector making production of $v$ particles rare at low energies

- Communicator's high mass

- Weak couplings....

- Production of v-particles may be observable at the LHC

- Some v-particles may be stable (dark matter candidates) and others decay to Standard Model particles 


\section{Hidden Valley \& Higgs Decays}

- V-pion is neutral pseudo-scalar

\section{Higgs decay to v-pions}

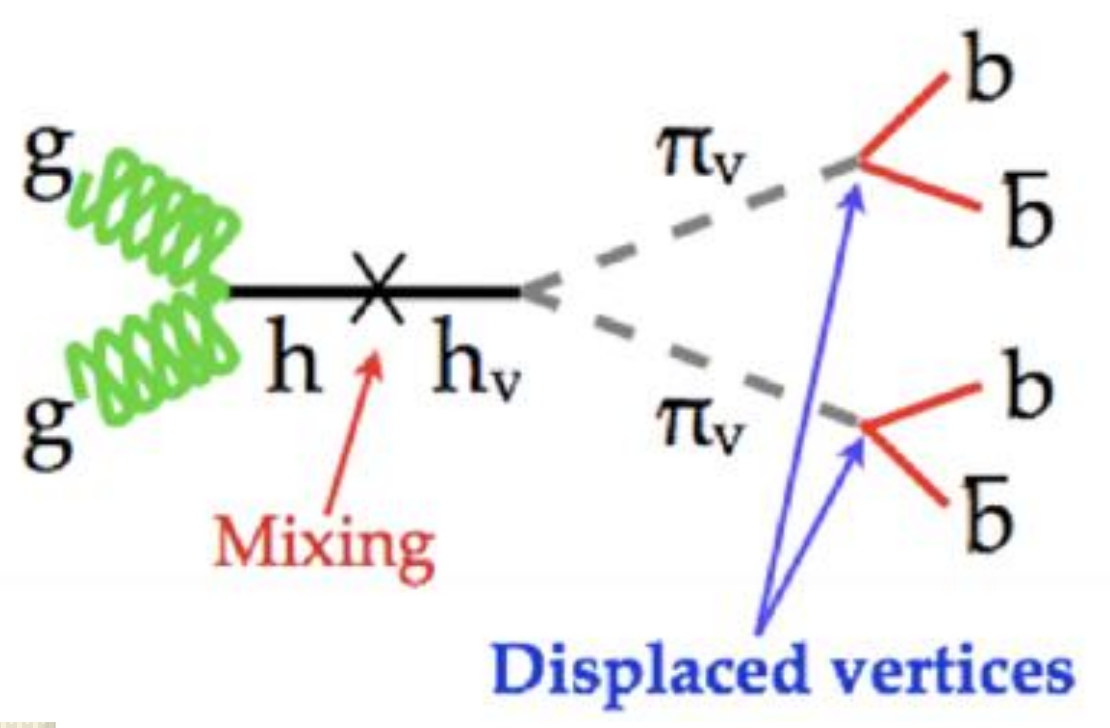

see also:

-M. Strassler \& K. Zurek, Phys Lett B 66 I (2008) 263-267

-S. Chang et al. arXiv:hep-ph/05 I I 250

-L. Carpenter et al. arXiv:hep-ph/0607204
- Displaced decay mainly to bottom quark

- We use 2 samples to study trigger strategies for this process:

- Ideal sample (signal only)

- Signal with pileup

- pileup for $L=10^{32} \mathrm{~cm}^{-2} \mathrm{~s}^{-1}$

- 4.I collisions/crossing

- 450ns bunch spacing

- Parameters:

- Higgs Mass $=140 \mathrm{GeV}$

- $\pi_{\mathrm{v}}$ Mass $=40 \mathrm{GeV}$

- $\pi_{\mathrm{v}} \mathrm{c \tau}=1500 \mathrm{~mm}$

- Events simulated using PYTHIA

- Work in collaboration with M. Strassler 


\section{Experimental Challenges}

- Neutral states decaying far from the interaction point lead to challenges for the trigger

- Current ATLAS triggers center on particles originating from the Interaction Point

- Long-lived Hidden Valley particles will decay throughout the detector volume

- Depending on where the decay occurs different approaches are required

- We need special triggers for each the signature produced in each system 


\section{ATLAS Trigger}

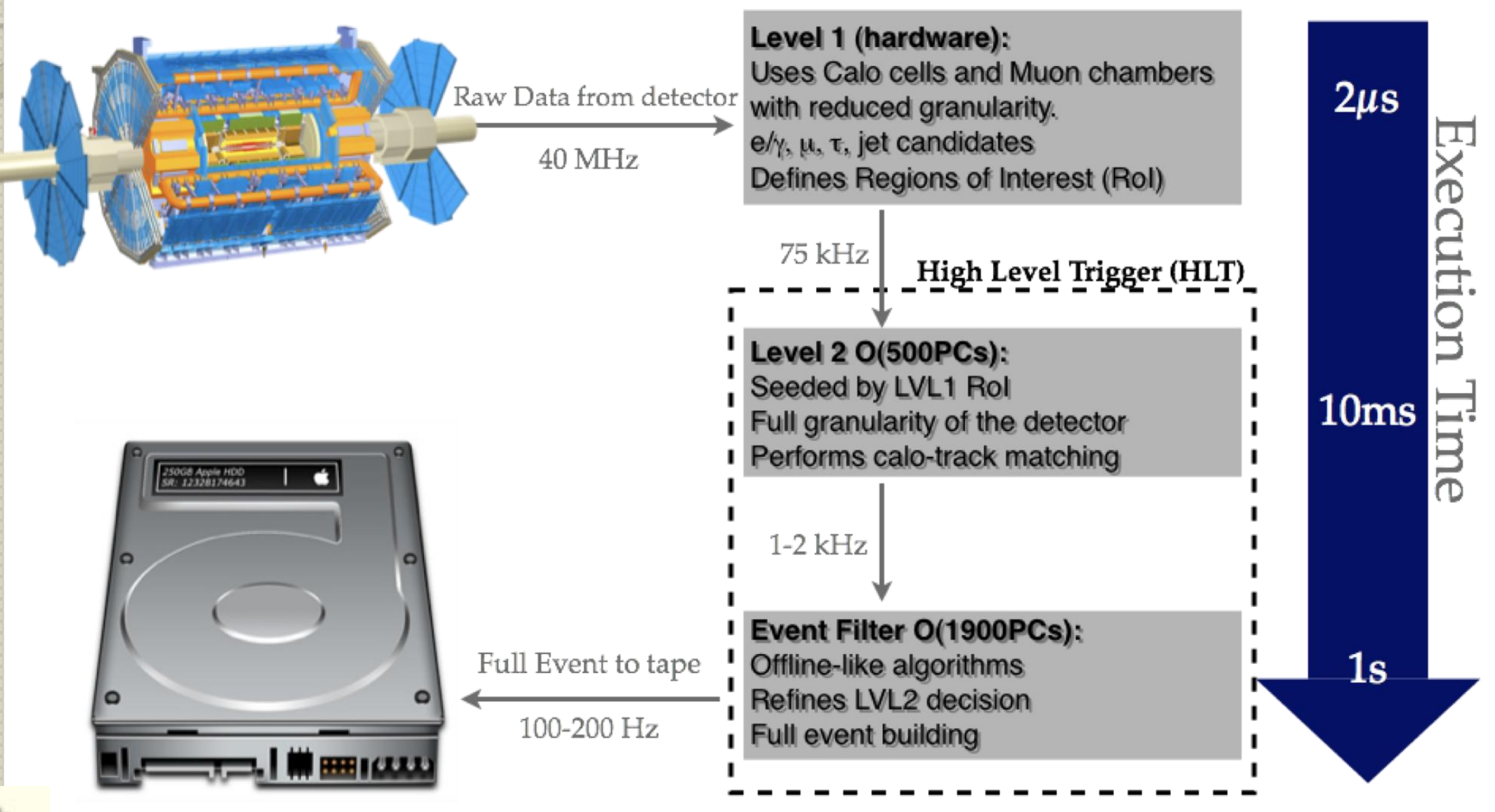




\section{Decays within ATLAS Detector}

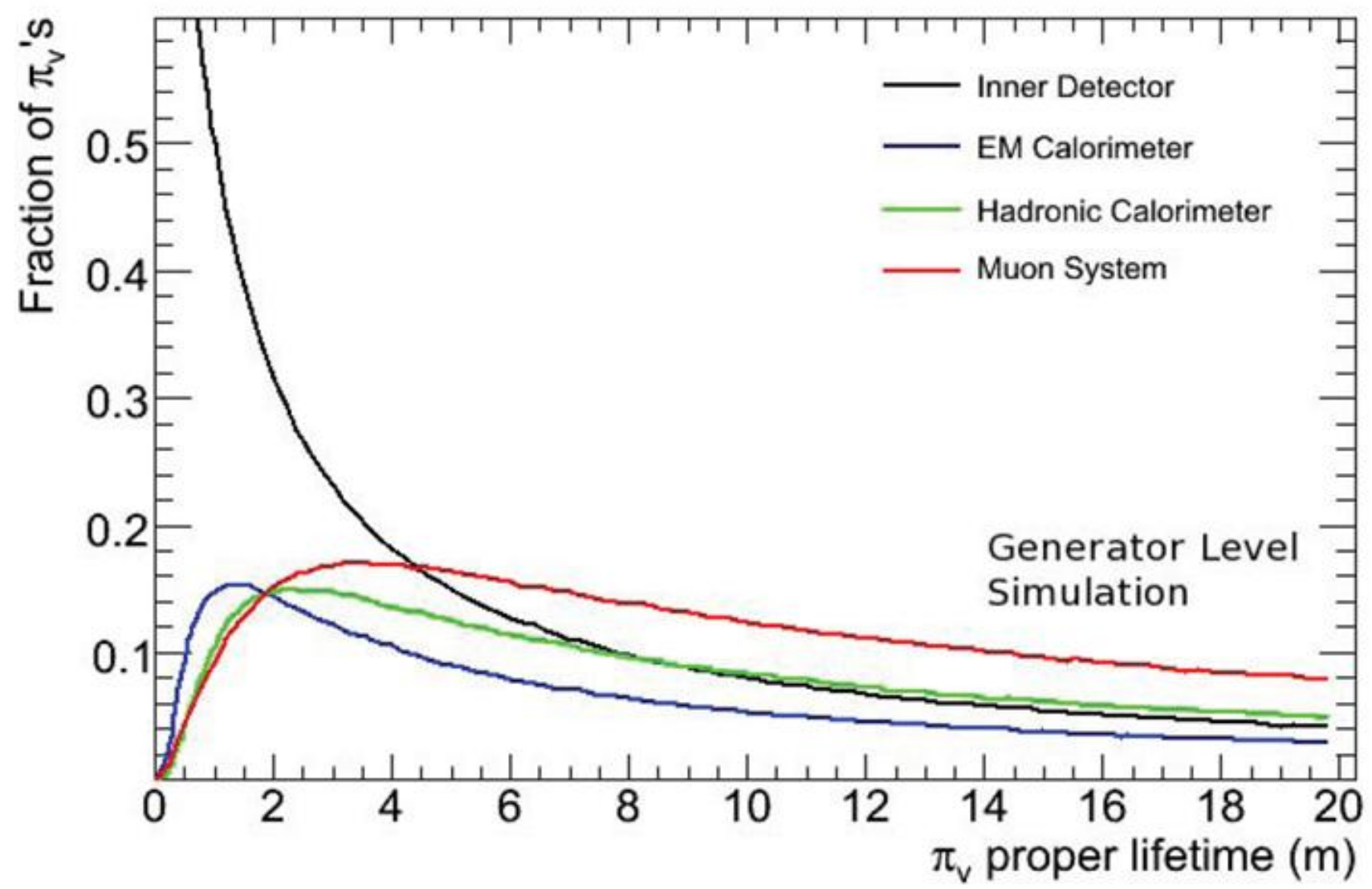

Probability for $\pi v$ from gg fusion to decay in each detector region vs ct for $|\eta|<2.5$ (Inner Detector coverage) 


\section{Decays in Muon Spectrometer}

- Little or no energy deposited in the calorimeter

- Characterized by a large number of charged tracks and a cluster of muon Regions of Interest (Rols)

- Only I muon reconstructed per muon Rol with standard trigger
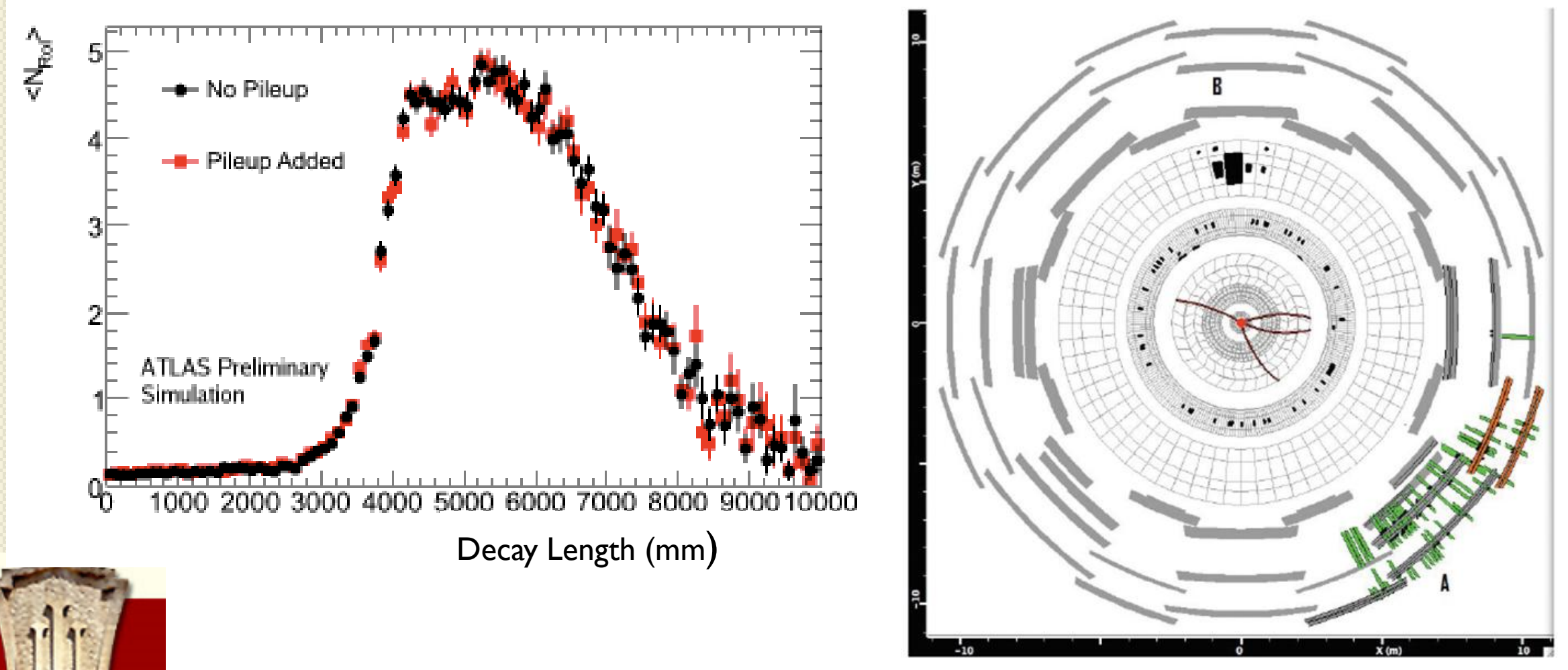


\section{Decays in Muon Spectrometer}

- Define a new Level 2 trigger algorithm using these signatures as:

- At least 3 muon Regions of Interest at Level I

- Isolation wrt jets and Inner Detector tracks

- $>70 \%$ Efficient for decays in the barrel Muon Spectrometer

- $>25 \%$ in the endcap region
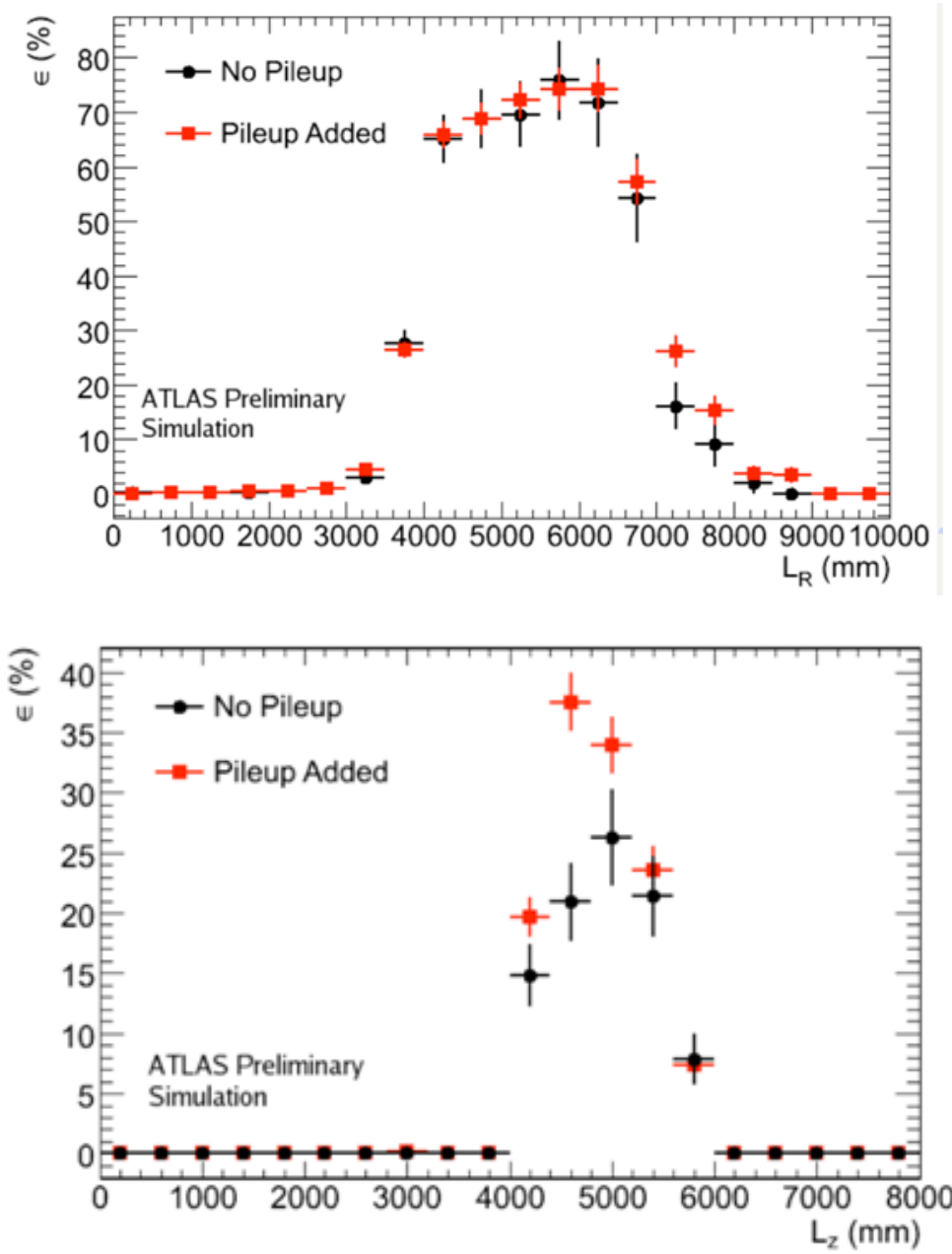


\section{Decays in the Hadronic Calorimeter}

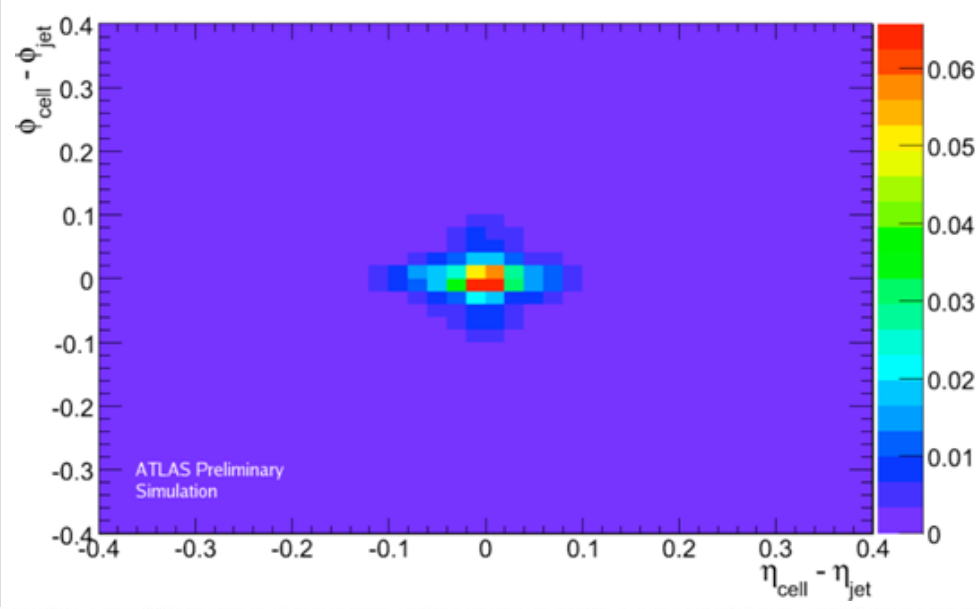

- Decays in the calorimeter produce very narrow jets

- No reconstructed tracks in the Inner Detector

- Large energy deposited in the Hadronic Calorimeter (HCAL )

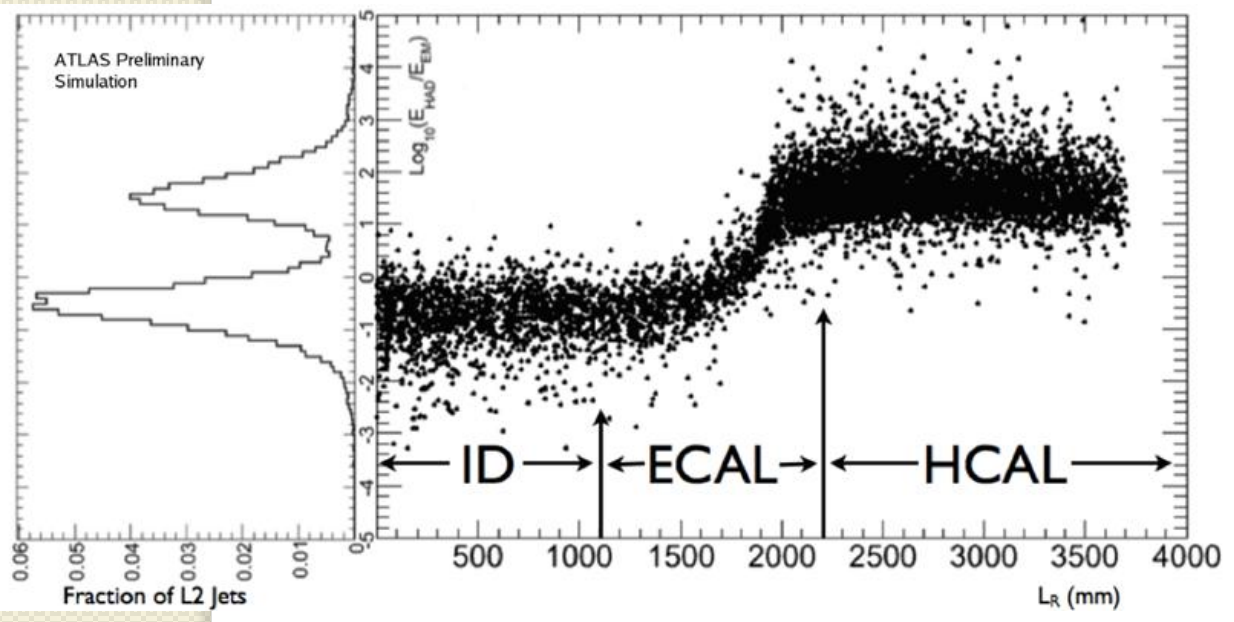

- Little energy in the Electromagnetic Calorimeter (ECAL)

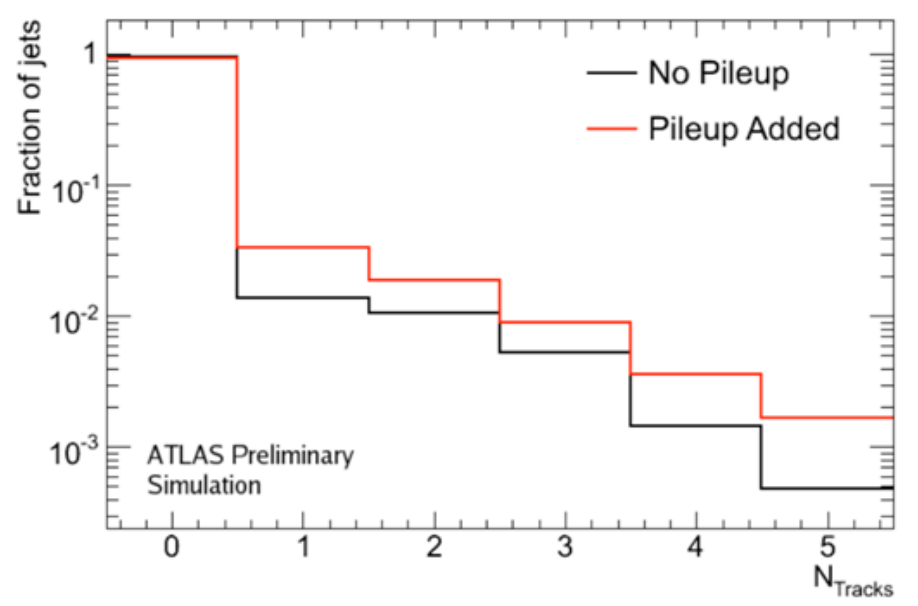




\section{Decays in the Hadronic Calorimeter}

- Narrow jet shape allows of use a Level-I $\tau$ trigger to select these decays

- We define a Level 2 trigger using these signatures as:

- $\log 10\left(\mathrm{E}_{\mathrm{HCAL}} / \mathrm{E}_{\mathrm{ECAL}}\right)>1$

- Isolation wrt Inner Detector tracks
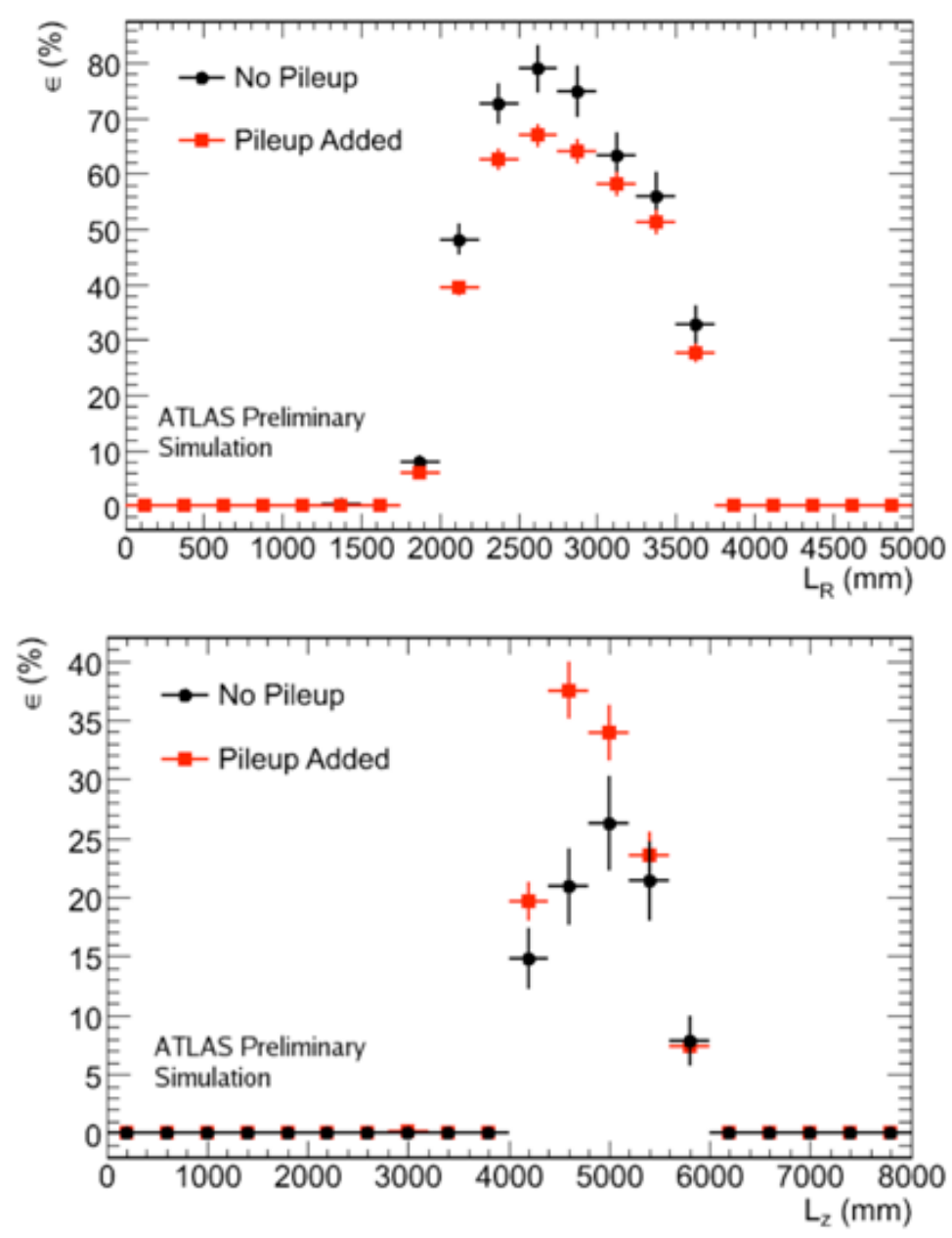


\section{Decays in the Inner Detector}
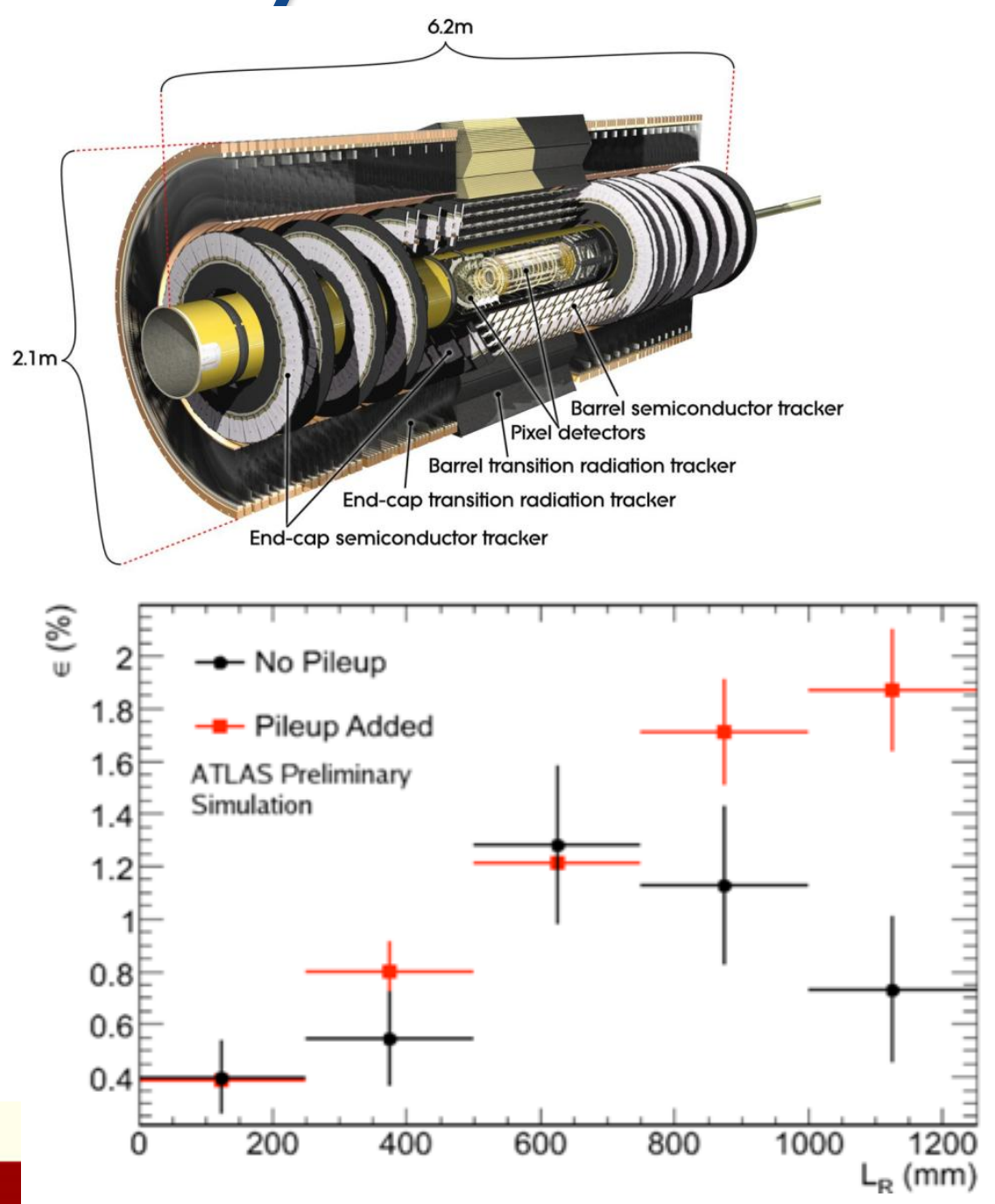

- Decays in the Inner Detector:

- low efficiency for normal tracking algorithms

- Trigger on trackless jets containing muons

- Level 2 jet trigger

- $E_{T} \geq 35 \mathrm{GeV}$ in Electrogmagnetic Calorimeter (ECAL)

- no reconstructed tracks

- Seed with Level Imuon

- Absolute efficiency 2\% (due to requiring the muon in the event)

- Studies ongoing to define a more efficient trigger in the Inner Detector

- Backtracking and vertex finding in ID

- jet substructure in the ECAL 


\section{Status of Improvements}

- New L2Trigger Algoritms

- Cluster of Muon objects isolated from tracks and jets

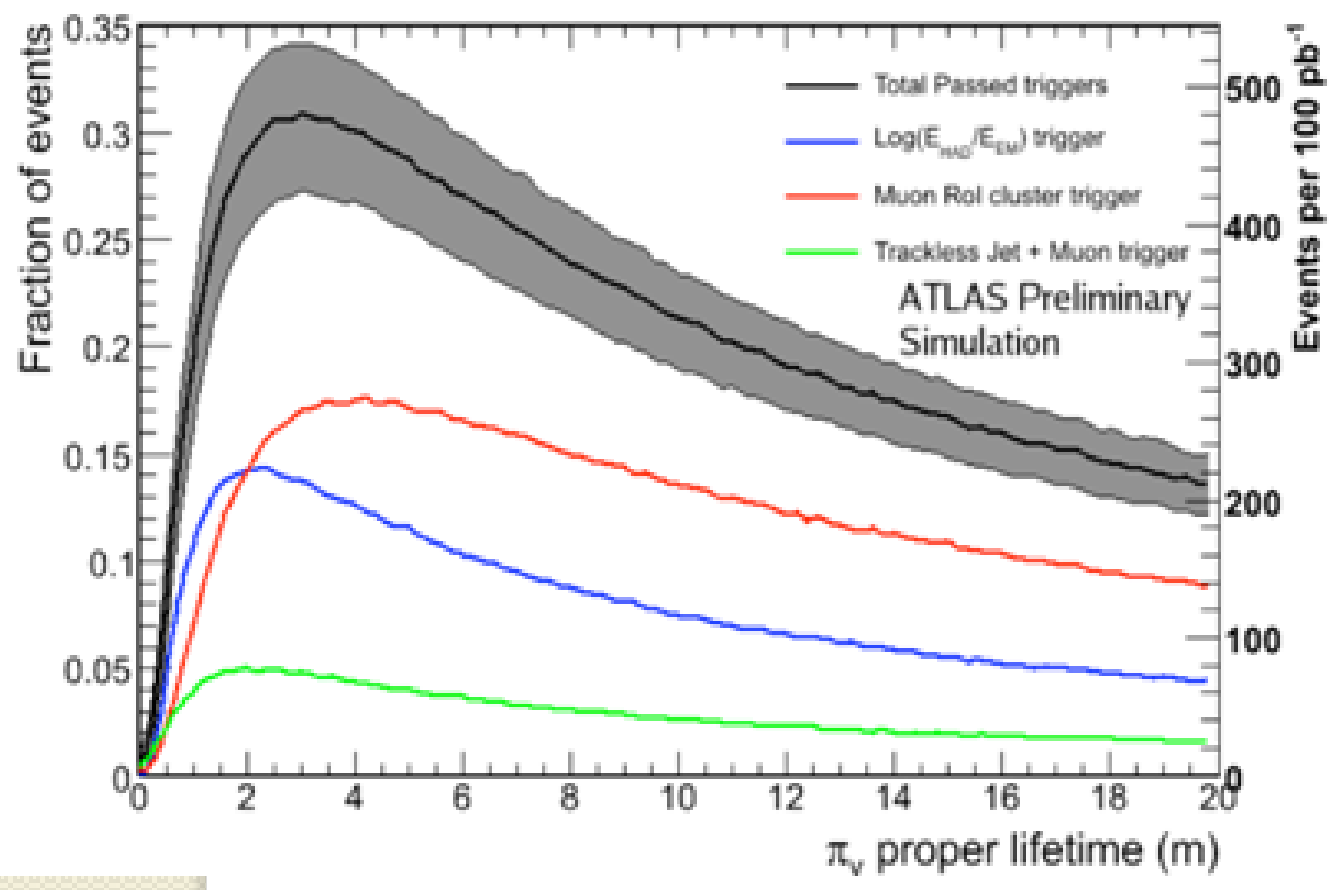

- LI dimuon trigger

- Narrow trackless jets with high hadronic/EM Energy

- LI tau trigger

- Trackless EM jet with a muon

- LI muon \& LI jet triggers

- Backgrounds

- None of 3M minbias events pass the triggers

- Sample of $10 \mathrm{TeV}$ di-jet events at $10^{32} \mathrm{~cm}^{-2} \mathrm{~s}^{-1}$ have less than I $\mathrm{Hz}$ combined L2 trigger rates 


\section{Related Searches}

- Ongoing work using similar strategies for neutral long-lived particles decaying to lepton jets (Weiner et al., Lin-Tao et al.)

- Trigger and reconstruction improvements for charged stable massive particles in each part of ATLAS

- Muon system (see talk by Shlomit Tarem)

- Calorimeters (see talk by Philippe Mermod)

- Ongoing work on Inner Detector 


\section{Conclusions}

- New signature-based triggers have been created to reconstruct long-lived neutral particles decaying in ATLAS

- Improvement in overall Higgs to Hidden Valley event efficiency from $\sim 2 \%$ to $>20 \%$

- Ongoing work on Inner Detector Decays and Event Filter trigger selection may contribute further improvements

- We are developing strategies to use ATLAS to search for neutral and charged massive long-lived particles in new physics models 